\title{
Technology assessment in radiation oncology: time for reassessment?
}

\author{
Jay S Loeffler
}

Within the field of radiation oncology, assessment of new technology has always been markedly different to that required to bring new cancer drugs to the market. Why? Foremost, the antineoplastic agent remains the sameionizing radiation; thus, predicting tumor control and treatment morbidity can be generally surmised on the basis of dose distribution to the target volume, dose distribution to normal structures within or nearby the target volume, total dose and time over which the radiation is delivered, and sensitivity of the tumor and the normal tissues exposed to radiation. The history of acceptance of new technology in radiation oncology such as port films, custom shielding, CT-based and MRI-based treatment planning, megavoltage beams, intensitymodulated delivery systems and proton therapy has been based on the general principles that the more accurate and conformal the radiation provided, the more favorable the patient outcome. Few would argue that these new technologies should provide better outcomes for our patients on the basis of improvement of dose distribution and accuracy of delivery. So why is it time for reassessment?

These new technologies in radiation oncology result in either new costs or higher costs or both to health-care systems. Obviously, given a limited budget for health-care provision, new costs for radiation oncology could mean that opportunities for advancement in other oncology activities such as cancer prevention initiatives (e.g. human papillomavirus vaccinations for children, smoking cessation programs) are lost. Rarely are new medical interventions associated with net savings and improved ....we have

to strongly

discourage

the potential

overuse

of these

techniques

simply for

the sake of

improved

reimbursement.

JS Loeffler is an Advisory Board member of Nature Clinical Practice Oncology.

\section{Competing interests}

The author declared no competing interests.

www.nature.com/clinicalpractice doi: $10.1038 /$ ncponc 1140 health-care outcomes. More commonly, when new technologies are found to be costeffective, other activities have to be abandoned to stay within budget. Since most societies are restrained by a relatively fixed health-care budget, cost-effectiveness analysis is needed for these new technologies even if 'perfect' data are not available from randomized clinical trials. If a fixed budget did not exist for health care, new radiation oncology technologies would be offered and readily accepted even for minimal benefits to patients.

I remain fiercely optimistic about our ability to continue the marvelous course of innovation in radiation oncology that we have all witnessed in the past 20 years. To successfully move forward, we will need to provide better information on clinical outcome so that we and others can critically evaluate these new technologies. As a medical discipline, we have to strongly discourage the potential overuse of these techniques simply for the sake of improved reimbursement, or because of patient or referring physician demands or provider ego. In addition, I think there are enormous opportunities for efficiency improvements that will leverage the incremental capital expenditures by allowing more patients to be treated during a fixed period of time. In our own experience, we have been able to reduce delivery time for patients receiving proton therapy by more than $50 \%$ over the past few years. 'Disruptive technology' will certainly emerge with time and will reduce the cost of radiotherapy. I predict that disruptive technology will have a similar impact on the discipline of radiation oncology as the digital camera has had on the photography market. 